\title{
Crystal structure of the C-terminal domain of the $\varepsilon$ subunit of human translation initiation factor elF2B
}

\author{
Jia Wei ${ }^{1,2}$, Minze $\mathrm{Jia}^{1}$, Cheng Zhang ${ }^{1}$, Mingzhu Wang ${ }^{1}$, Feng Gao ${ }^{1}$, Hang Xu ${ }^{1 凶}$, Weimin Gong ${ }^{1 凶}$ \\ ${ }^{1}$ National Laboratory of Biomacromolecules, Institute of Biophysics, Chinese Academy of Sciences, Beijing 100101, China \\ ${ }^{2}$ College of Life Sciences, Graduate University of Chinese Academy of Sciences, Beijing 100049, China \\ $\bowtie$ Correspondence: wgong@ibp.ac.cn (W. Gong), hxu@moon.ibp.ac.cn (H. Xu) \\ Received April 30, 2010 Accepted May 25, 2010
}

\begin{abstract}
Eukaryotic translation initiation factor elF2B, the guanine nucleotide exchange factor (GEF) for elF2, catalyzes conversion of elF2.GDP to elF2.GTP. The elF2B is composed of five subunits, $\alpha, \beta, y, \delta$ and $\varepsilon$, within which the $\varepsilon$ subunit is responsible for catalyzing the guanine exchange reaction. Here we present the crystal structure of the C-terminal domain of human elF2B $\varepsilon$ (eIF2B $\varepsilon-C T D$ ) at 2.0-A resolution. The structure resembles a HEAT motif and three charge-rich areas on its surface can be identified. When compared to yeast elF2B $\varepsilon$-CTD, one area involves highly conserved AA boxes while the other two are only partially conserved. In addition, the previously reported mutations in human elF2B $\varepsilon$-CTD, which are related to the loss of the GEF activity and human VWM disease, have been discussed. Based on the structure, most of such mutations tend to destabilize the HEAT motif.
\end{abstract}

KEYWORDS eukaryotic translation initiation factor 2B (elF2B), guanine nucleotide exchange factor (GEF), crystal structure, HEAT motif, vanishing white matter (VWM)

\section{INTRODUCTION}

The formation of the Met-tRNA $A_{i}^{\text {Met }}$ elF2 $\cdot$ GTP ternary complex results in the 43S pre-initiation complex (PIC) and is essential for cap-dependent translation initiation in eukaryote (Kapp and Lorsch, 2004; Marintchev and Wagner, 2004; Sonenberg and Hinnebusch, 2007). Because this ternary complex formation requires GTP-bound elF2, elF2.GDP can not enter the initiation cycle until GDP is exchanged to GTP.
Such exchange is catalyzed by elF2B and known as one of the rate limiting steps in translation initiation (Pavitt, 2005). It has been found that even little disturbance in elF2B function may cause severe problems. For example, mutations of elF2B have been identified in an autosomal recessive neurodegenerative disease, known as VWM/CACH (vanishing white matter or childhood ataxia with central nervous system hypomyelination) (Li et al., 2004; Fogli and Boespflug-Tanguy, 2006; Scali et al., 2006; Maletkovic et al., 2008).

The elF2B consists of five subunits $a-\varepsilon$, which can be divided to two subcomplexes according to their functions (Cigan et al., 1993; Pavitt et al., 1998; Williams et al., 2001a, b; Pavitt, 2005; Mohammad-Qureshi et al., 2007a). The subunits $\alpha, \beta$ and $\delta$ form a regulatory subcomplex, which responds to the phosphorylation of elF2 $\alpha$ and cellular stresses (Pavitt et al., 1998; Krishnamoorthy et al., 2001; Williams et al., 2001a; Dever, 2002; Hinnebusch, 2005; Smirnova et al., 2005). Another subcomplex is catalysis relevant, containing subunits $\gamma$ and $\varepsilon$. The subunit $\varepsilon$ is responsible for the guanine nucleotide exchange reaction, and can be regulated by direct phosphorylation (Wang et al., 2001; Wang and Proud, 2008). Interestingly, the C-terminal fragment of yeast elF2B $\varepsilon$ (residues 518-712) is sufficient to catalyze the guanine nucleotide exchange of elF2 (Pavitt et al., 1998; Gomez et al., 2002; Mohammad-Qureshi et al., 2007b).

The elF2B $\varepsilon$-CTD not only covers the minimal catalytic domain, but also contains highly conserved regions for interaction with other proteins. Two AA boxes (rich in acidic and aromatic residues) in elF2B $\varepsilon$-CTD (Asano et al., 1999; Boesen et al., 2004) are also conserved in elF5 and elF4G (Marcotrigiano et al., 2001; Bieniossek et al., 2006; Wei et al., 
2006). elF2B $\varepsilon$ and elF5 employ these AA boxes to bind lysine-rich K boxes in elF2 $\beta$ (Das et al., 1997; Asano et al., 1999; Das and Maitra, 2000; Singh et al., 2006). In addition to the conserved sequence at $\mathrm{AA}$ boxes, similar structural folding known as HEAT motif is observed in the C-terminal domains of elF2Br, elF5 and elF4G.

In this study, the $2.0-\AA$ crystal structure of the C-terminal domain (residues 547-721) of human elF2B $\varepsilon$ was determined, which has been the best model of human elF2BE-CTD with the highest resolution. The HEAT motif in this structure is similar to that in its yeast homolog, but they have low sequence identity and differ in the arrangement of their internal helices as well as in surface electrostatic potential. Finally, the reported mutations of human elF2B $\varepsilon$-CTD related to catalytic activity and $\mathrm{VWM} / \mathrm{CACH}$ are discussed based on the structure.

\section{RESULTS}

\section{Overall structure}

The crystal structure of human elF2BE-CTD has been solved at 2.0-A resolution with MAD method and refined to $R_{\text {work }}$ and $R_{\text {free }}$ of $20.4 \%$ and $25.5 \%$, respectively (Table 1 ). Similar to its homolog in yeast (PDB ID: 1PAQ) (Boesen et al., 2004), the human elF2B $\varepsilon$-CTD structure is highly helical, containing nine $\alpha$-helices ( $\alpha 1-9)$ and two $31_{10}$-helices ( $\eta 1$ and $\left.\eta 2\right)$ (Fig. 1). These helices are divided into four layers of helical repeats, each containing one anti-parallel helical pair. These helical pairs are packed along the axis that is perpendicular to the helices, while left-handed twisting occurs between adjacent layers. By such manner, human elF2B $\varepsilon$-CTD is assembled into a globular domain with approximate dimensions of $45 \times$ $40 \times 30 \AA^{3}$, which is widely recognized as HEAT motif and has been reported to function in protein interactions (Marcotrigiano et al., 2001; Bieniossek et al., 2006; Wei et al., 2006). In addition, helices $\alpha 6, \alpha 7$ and $\alpha 9$ constitute two AA boxes (Fig. 2), which are highly conserved and required for the interaction with elF2 $\beta \mathrm{N}$-terminal lysine-rich portion (Asano et al., 1999).

\section{Structural comparison of the elF2B $\varepsilon$-CTDs from human and yeast}

Although the overall folding of human elF2BE-CTD is similar to that of yeast elF2Be-CTD (PDB ID: 1PAQ) (Boesen et al., 2004) (154 Ca's aligned with RMSD of $2.3 \AA$ by using DALI) (Holm and Park, 2000), these domains exhibit different structural features due to their low sequence identity $(20 \%)$. First, the bending angles in individual helices show significant variation in human and yeast elF2B $\varepsilon$-CTD. An extreme example is demonstrated at the last helical repeat in human elF2B $\varepsilon$-CTD, where the bending degree is so high that an extra helix $(\alpha 8)$ is formed to adapt to such bending conformation (Fig. 1 and Fig. 2). In addition, the conformation of the loop linkage between $\alpha 3$ and $\alpha 4$ is quite different (Fig. 3A). The $\alpha 3 / \eta 1$ helix in human elF2B $\varepsilon$-CTD terminates earlier than its yeast counterpart ( $\alpha 3)$ and thus releases two end residues into loop conformation (Fig. 2 and Fig. 3A). Finally, both elF2B $\varepsilon$-CTD domains contain two $3_{10}$-helices that form natural extension of the adjacent alpha helices. In human elF2B $\varepsilon$-CTD, the first $3_{10}$-helix $(\eta 1)$ is found at the Cterminus of $\alpha 3$ and the second $(\eta 2)$ is at the $N$ terminus of $\alpha 6$. However, in yeast, the two $33_{10}$-helices are accommodated at the $\mathrm{C}$-terminus of $\alpha 4$ and at the $\mathrm{N}$-terminus of $\alpha 8$, respectively (Fig. 2).

\section{Surface property of human eIF2B $\varepsilon-C T D$}

The surface of human elF2B $\varepsilon$-CTD contains three areas with condensed charge, two acidic and one basic (Fig. 3B). The first area (area I) is acidic and formed by residues Glu591, Glu602, Glu644, Asp651, Glu655, Glu657, Asp689 and Asp692. Area II includes five acidic residues (Glu674, Glu678, Glu679, Glu712 and Glu714) and two aromatic residues (Tyr671 and Trp709), all of which are involved in the two AA boxes (Fig. 2 and Fig. 3B). Composed of Lys626, Lys665, Gln702 and Arg705, area III is positively charged and located at the opposite side to the area I. The results of sequence alignment of eukaryotic elF2BE-CTD's (Fig. 2) demonstrate: all the above areas and residues therein are highly conserved among mammalian species; area II is mostly conserved among all species, suggesting a common interaction mode between eIF2B $\varepsilon$ AA boxes and elF2 $\beta$; area I and III are only of limited similarity (Fig. 2, Fig. 3B and 3 C), likely to underlie various regulatory mechanisms.

\section{DISCUSSION}

\section{Structural implication of the mutation causing the loss of the GEF activity of elF2B}

It has been reported that Glu577Ala mutant of human elF2B $\varepsilon$ displays less catalytic activity and exhibits reduced binding affinity for elF2 (Wang and Proud, 2008). This is consistent with the hypothesis that the corresponding residue in yeast elF2B $\varepsilon$, Glu569, is critical in interacting with elF2 $\gamma$ and catalyzing GDP-GTP exchange (Mohammad-Qureshi et al., 2008; Wang and Proud, 2008). In human elF2BE-CTD, Glu577 participates in a hydrogen/salt bonding network with Thr560 and Arg563 (Fig. 4A). As residues Glu577, Thr560 and Arg563 are highly conserved (Fig. 2) and a similar bonding network was identified in yeast elF2B $\varepsilon-C T D$ (Fig. 4B), the interactions between these residues are likely to play an important role in elF2Be-catalyzed nucleotide exchange. One obvious consequence of the above interactions is the protection and stabilization of Glu577 in the absence of elF2 $\gamma$, while further investigation is required for 
Table 1 Statistics of data reduction and structure refinement

\begin{tabular}{|c|c|c|c|c|}
\hline \multirow{2}{*}{$\begin{array}{l}\text { MAD data collection } \\
\text { space group }\end{array}$} & inflection & peak & remote & \multirow{2}{*}{$\begin{array}{c}\text { refinement } \\
\text { C222 }\end{array}$} \\
\hline & \multicolumn{3}{|c|}{$C 222_{1}$} & \\
\hline unit cell parameters & \multicolumn{3}{|c|}{$a=46.5 \AA, b=66.0 \AA, c=136.5 \AA$} & $\begin{array}{c}a=46.5 \AA, b=66.1 \AA, \\
c=136.1 \AA\end{array}$ \\
\hline wavelength $(\AA)$ & 0.9796 & 0.9794 & 0.9600 & 0.9794 \\
\hline resolution range $(\AA)^{a}$ & $\begin{array}{c}50-2.3 \\
(2.38-2.30)\end{array}$ & $\begin{array}{c}50-2.3 \\
(2.38-2.30)\end{array}$ & $\begin{array}{c}50-2.3 \\
(2.38-2.30)\end{array}$ & $\begin{array}{c}50-2.0 \\
(2.07-2.00)\end{array}$ \\
\hline No. of total reflections & 69,384 & 69,256 & 69,370 & 95,130 \\
\hline $\begin{array}{l}\text { No. of unique reflec- } \\
\text { tions }\end{array}$ & 9770 & 9770 & 9719 & 13,260 \\
\hline average redundancy & $7.1(6.8)$ & $7.1(6.8)$ & $7.1(7.2)$ & $7.2(7.4)$ \\
\hline completeness (\%) & $99.6(100.0)$ & $99.6(100.0)$ & $99.5(100.0)$ & $90.6(84.1)$ \\
\hline$R_{\text {merge }}{ }^{\mathrm{b}}$ & $0.057(0.155)$ & $0.072(0.170)$ & $0.062(0.151)$ & $0.070(0.313)$ \\
\hline$<I>I<\sigma(I)>$ & $34.8(11.1)$ & $28.2(9.8)$ & $31.3(11.6)$ & $24.1(4.8)$ \\
\hline \multicolumn{5}{|l|}{ refinement statistics } \\
\hline space group & \multicolumn{4}{|c|}{$C 222_{1}$} \\
\hline unit cell parameters & \multicolumn{4}{|c|}{$a=46.5 \AA, b=66.1 \AA, c=136.1 \AA$} \\
\hline$R_{\text {work }} / R_{\text {free }}(\%)^{\mathrm{c}}$ & \multicolumn{4}{|c|}{$20.4 / 25.5$} \\
\hline r.m.s.d. bond length $(\AA$ & \multicolumn{4}{|c|}{0.0095} \\
\hline r.m.s.d. bond angle $\left({ }^{\circ}\right)$ & \multicolumn{4}{|c|}{1.18} \\
\hline No. of protein residues & \multicolumn{4}{|c|}{174} \\
\hline No. of water molecule & \multicolumn{4}{|c|}{144} \\
\hline No. of glycerol molecu & \multicolumn{4}{|c|}{1} \\
\hline \multicolumn{5}{|c|}{ average temperature factor $\left(\AA^{2}\right)$} \\
\hline \multicolumn{2}{|c|}{ protein main-chain atoms } & \multicolumn{3}{|c|}{24.4} \\
\hline \multicolumn{2}{|c|}{ protein side-chain atoms } & \multicolumn{3}{|c|}{25.8} \\
\hline water molecules & & \multicolumn{3}{|c|}{27.6} \\
\hline glycerol molecule & & \multicolumn{3}{|c|}{17.8} \\
\hline \multicolumn{5}{|l|}{ ramachandran plot (\%) } \\
\hline \multicolumn{2}{|c|}{ residues in most favored regions } & \multicolumn{3}{|c|}{97.5} \\
\hline \multicolumn{2}{|c|}{ residues in additionally allowed regions } & \multicolumn{3}{|c|}{2.5} \\
\hline \multicolumn{2}{|c|}{ residues in generously allowed regions } & \multicolumn{3}{|c|}{0} \\
\hline \multicolumn{2}{|c|}{ residues in disallowed regions } & \multicolumn{3}{|c|}{0} \\
\hline
\end{tabular}

${ }^{a}$ Data for the highest resolution bin is in parentheses.

${ }^{\mathrm{b}} R_{\text {merge }}=\sum|\mathrm{li}-/ \mathrm{m}| / \sum \mathrm{li}$, where $/ \mathrm{i}$ is the intensity of the measured reflection and $/ \mathrm{m}$ is the mean intensity of all symmetry-related reflections.

${ }^{\mathrm{c}} R_{\text {work }}=\sum|| F_{\text {obs }}|-| F_{\text {calc }}|||\Sigma| F_{\text {obs }} \mid$, where $F_{\text {obs }}$ and $F_{\text {calc }}$ are observed and calculated structure factors, respectively. $R_{\text {free }}=\sum \mathrm{T}|| F_{\text {obs }}|-| F_{\text {calc }} \mid$ $\left|/ \Sigma_{\mathrm{T}}\right| F_{\text {obs }} \mid$, where $T$ denotes a test data set of about $5 \%$ of the total reflections randomly chosen and set aside prior to refinement.

thorough and better understanding.

\section{Structural implication of the mutations causing the human disease VWM/CASH}

Up to date, several mutations in human elF2BE-CTD (residues 547-721) have been assigned with $\mathrm{VWM} / \mathrm{CACH}$, including Pro604Ser, Leu605frameshift, Trp628Arg,
Trp628stop, Ile649Thr, Glu650Lys, Lys665-Tyr671 deletion, and Ser610-Asp613 deletion (Fogli and Boespflug-Tanguy, 2006; Scali et al., 2006; Maletkovic et al., 2008; Wu et al., 2009).

Deletion of four residues Ser610-Asp613 in human elF2BE-CTD was recently reported in a Chinese VWM patient (Wu et al., 2009). These four residues are located on the loop between helices $\alpha 3$ and $\alpha 4$ (Fig. 4C), which displays different 

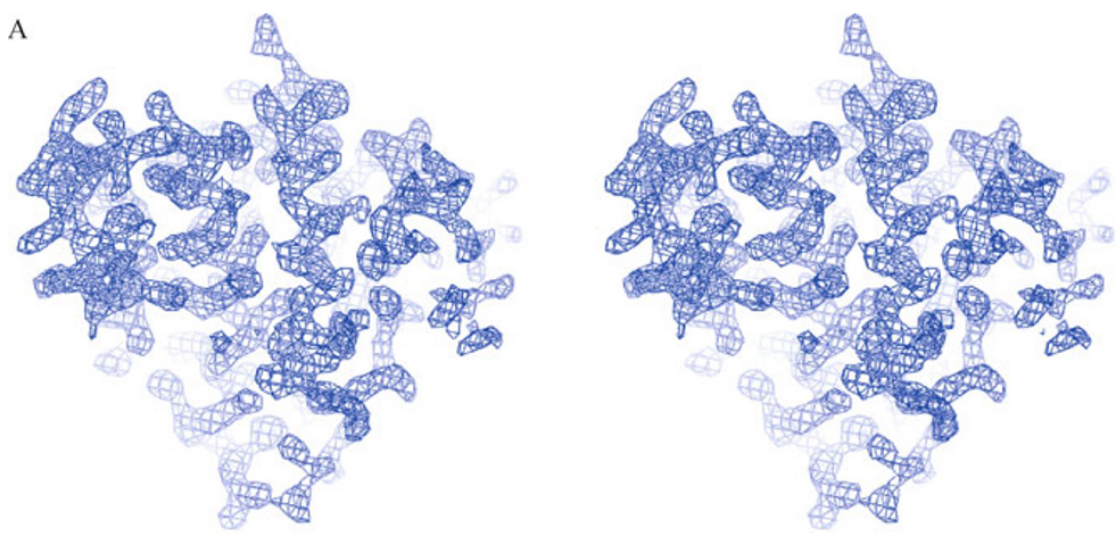

B
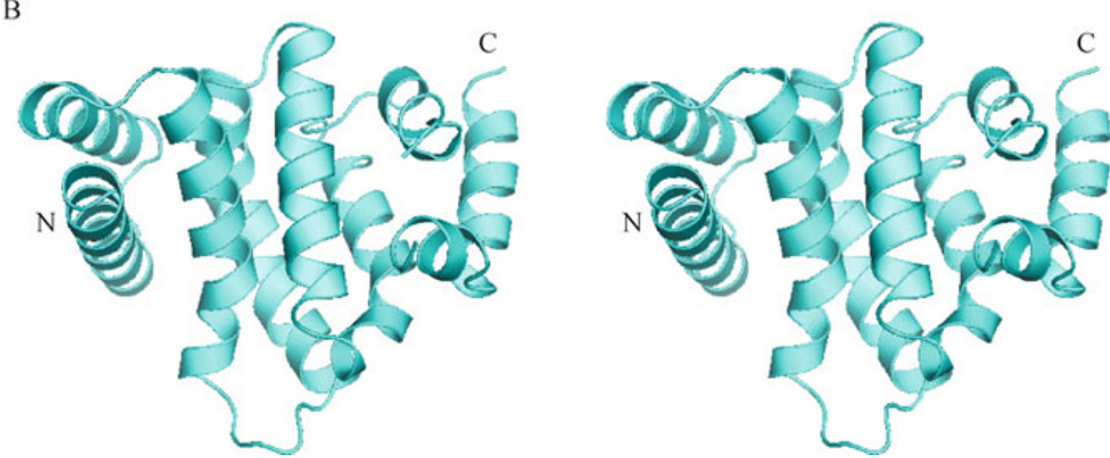

Figure 1. The overall structure of human eIF2BE-CTD (stereo view). (A) Experimental electron density map based on the MAD phases after density modification (stereo view). The map is contoured at $1.5 \sigma$. (B) Stereoview of the overall structure of human elF2B $\varepsilon$-CTD.

conformations in human and yeast elF2B $\varepsilon$-CTD as described above (Fig. 3A). With the loop shortened, the orientation of flanking helices $\alpha 3 / \eta 1$ and $\alpha 4$ might be forced to alter. This would consequently change the packing at the core of HEAT domain. In addition, this loop was suggested to play an important role in catalysis, based on the comparisons with other guanine nucleotide exchange factors (Boesen et al., 2004). Deletion of the residues Lys665-Tyr671 would highly reduce the stability of protein folding, since these residues are located in a6 (Fig. 4C), a core helix in HEAT repeat packing. Mutation of Trp628stop resulting in the lack of the two important AA boxes, as well as mutation of Leu605frameshift, would destroy the integral structure of elF2B $\varepsilon-C T D$.

In the rest point mutations, Pro604Ser, Trp628Arg and lle649Thr are identified in hydrophobic cores of human elF2BE-CTD. Residue Pro604 is involved in hydrophobic interactions with Tyr617, Leu621 and Leu624 (Fig. 4D), while Trp628 with Leu561, Phe603 and Leu624 (Fig. 4E), and lle649 with Leu601, Leu646, Phe652, Phe653, Leu667, Phe670 and Leu676 (Fig. 4F). As polar residues were introduced by Pro604Ser, or Trp628Arg, or lle649Thr, the hydrophobic interaction would be highly weakened. In contrast to the above three mutations, the environment around Glu650 is hydrophilic and it forms salt bond with Arg698 plus hydrogen bond with Trp684. These interactions stabilize the packing of helices $\alpha 5, \alpha 8, \alpha 7$ and the loop after a7 (Fig. 4G). Mutation of Glu650Lys would disturb such bonding network and therefore destabilize the HEAT folding.

Based on the above analysis, most VWM related mutations within human elF2BE-CTD are more likely to associate with structural integrity and stability, rather than the catalytic activity.

\section{MATERIALS AND METHODS}

Plasmid construction, protein expression and purification

The gene fragment of human elF2Be-CTD (residues 547-721) was amplified from a pDEST566 vector containing full-length elF2B $\varepsilon$ gene (a generous gift from Dr. James Zhijie Liu, Institute of Biophysics, Chinese Academy of Sciences), and constructed into p28 vector for expression. The above construct contained an incidental mutation Glu678Gly and N-terminal (His)6 tag. The plasmid was transferred into methionine-auxotrophic $E$. coli strain b834 and cells were grown in $\mathrm{M} 9$ media containing $40 \mathrm{mg} / \mathrm{L}$ selenomethionine, $2 \mathrm{mM} \mathrm{MgSO}_{4}$, $0.1 \mathrm{mM} \mathrm{CaCl} 2$ and $0.05 \mu \mathrm{M} \mathrm{FeSO}$, with kanamycin sulfate $(50 \mu \mathrm{g} / \mathrm{mL})$ at $37^{\circ} \mathrm{C}$. Induction was performed by $0.25 \mathrm{mM} \mathrm{IPTG}$ for 3 h. Cells were lysed by sonication in $20 \mathrm{mM}$ Tris-HCl, pH 7.7, $200 \mathrm{mM}$ 

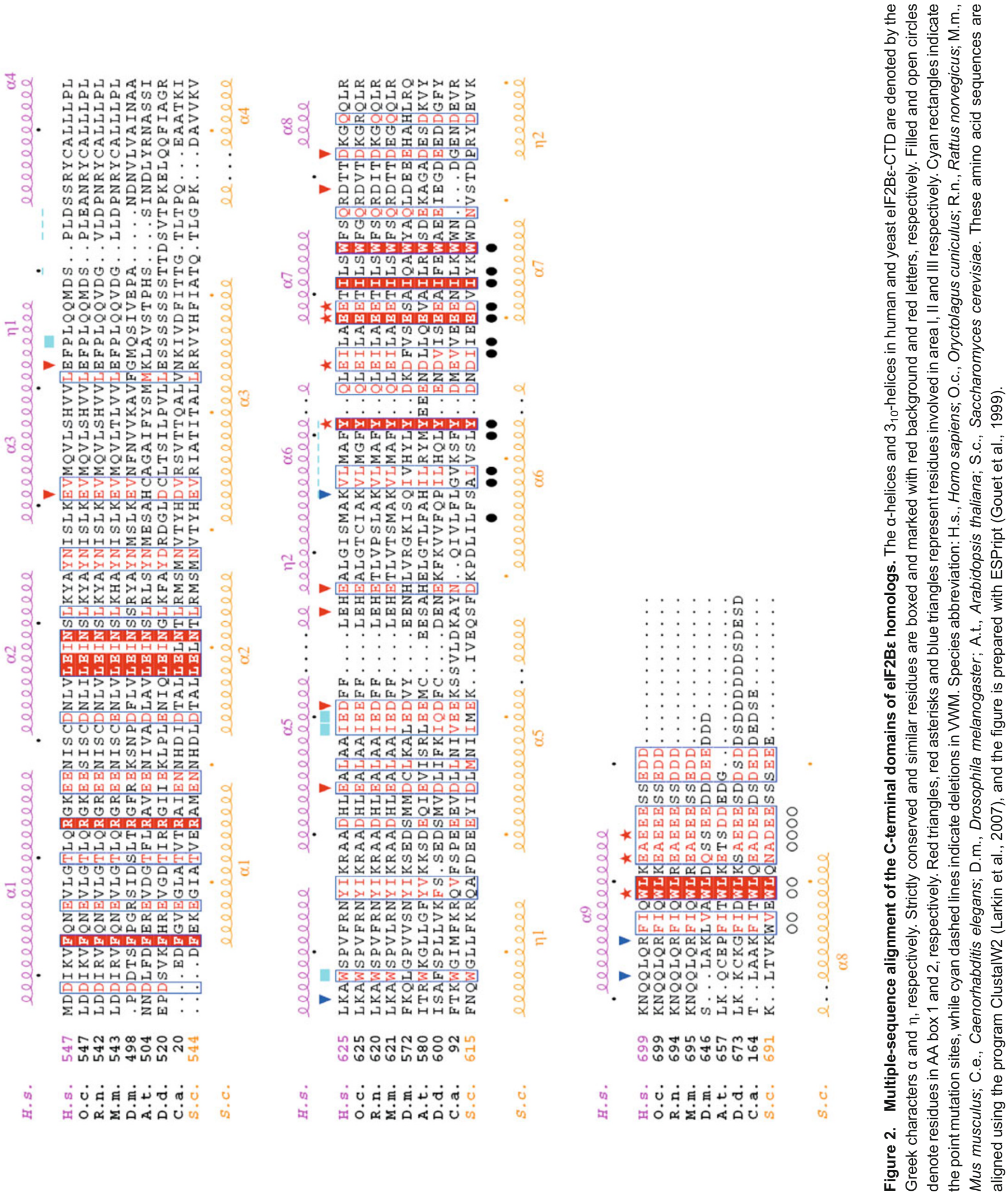

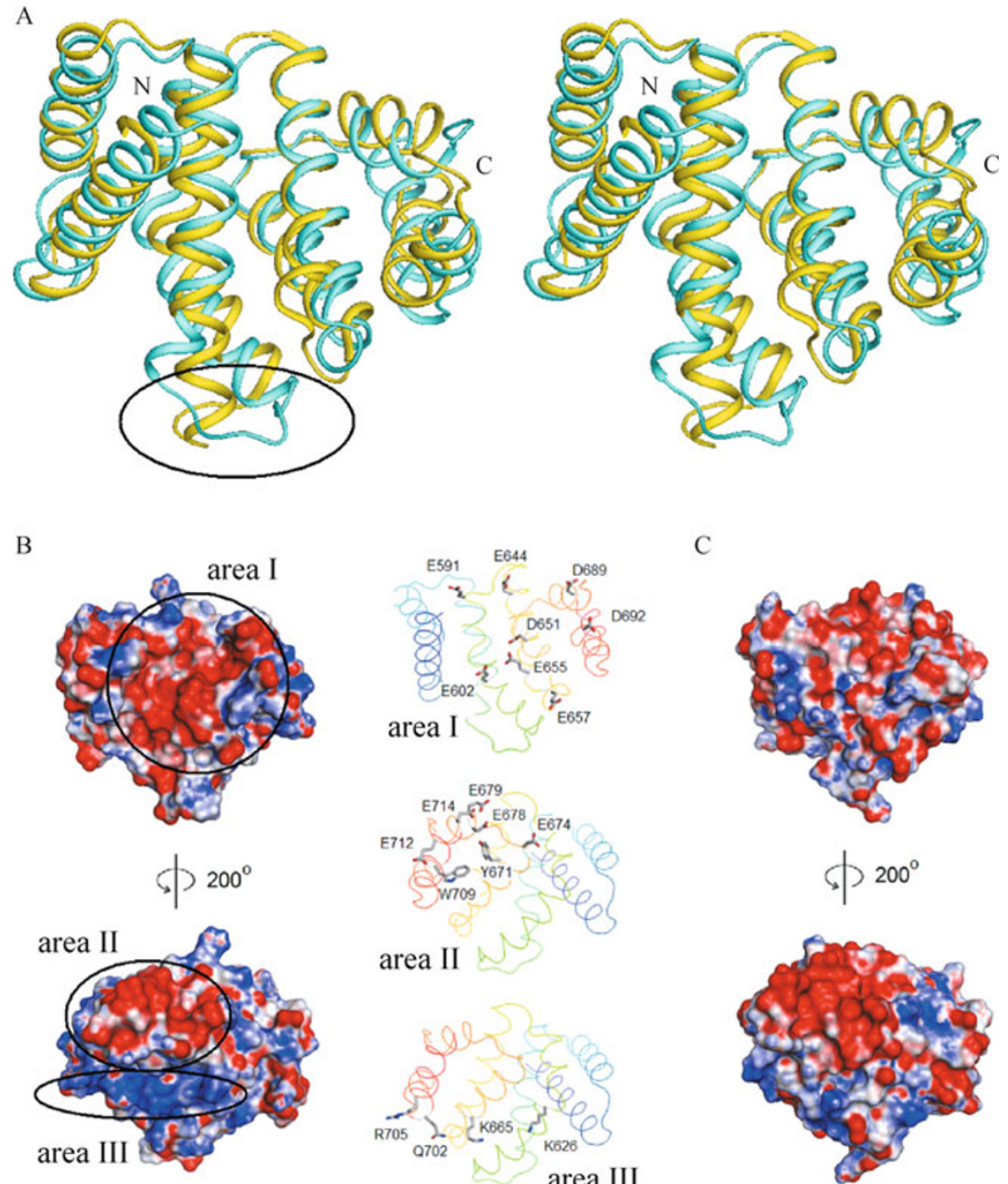

C
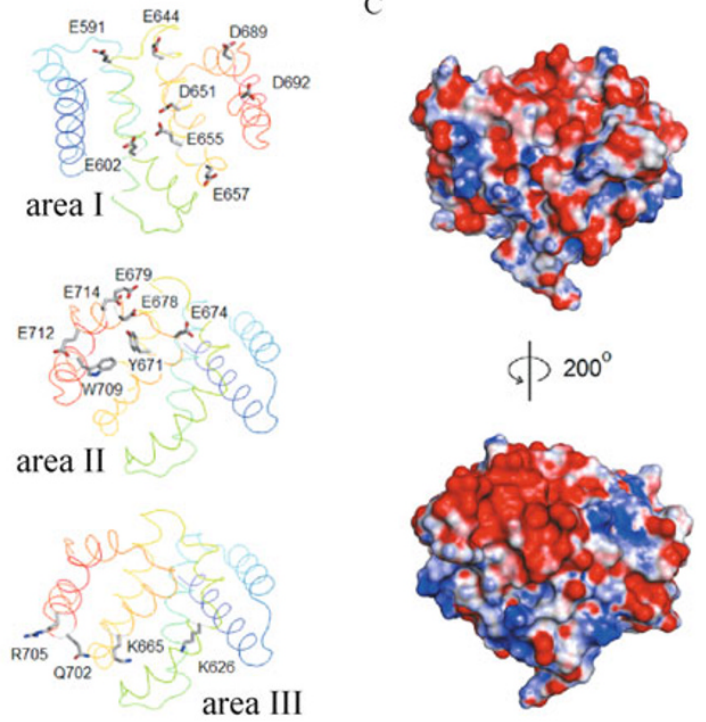

human $\mathrm{elF} 2 \mathrm{~B} \varepsilon-\mathrm{CTD}$

yeast elF2B $\varepsilon-C T D$

Figure 3. Structural comparison of human and yeast elF2B $\varepsilon$-CTD. (A) Structural alignment of C-termini of human and yeast elF2B $\varepsilon$ (stereo view). Human and yeast elF2Be-CTD are shown in cyan and yellow, respectively. The loops with different conformation in two structures are circled. (B) Electrostatic potential distribution on the surface of human elF2B $\varepsilon$-CTD in different views. Charge-rich areas I, II and III are circled. Residues involved in area I, II and III are demonstrated with their side chains, respectively. (C) Electrostatic potential distribution on the surface of yeast elF2BE-CTD (PDB ID: 1PAQ), shown as the same orientation in $(B)$.

$\mathrm{NaCl}, 20 \mathrm{mM}$ Imidazole, and $1 \mathrm{mM}$ PMSF. The recombinant protein in lysate was purified by affinity chromatography with a Nickel Chelating Sepharose $^{\mathrm{TM}}$ Fast Flow column (GE), followed by gel filtration chromatography with a Superdex75 column (GE).

\section{Crystallization and structure determination}

Crystals of selenomethionyl human elF2BE-CTD were obtained by hanging-drop vapor-diffusion method plus streak-seeding using a feline whisker at $16^{\circ} \mathrm{C}$ within one week. Hanging drops were prepared by mixing a solution $(1 \mu \mathrm{L})$ containing human elF2B $-\mathrm{CTD}(19 \mathrm{mg} / \mathrm{mL}$ protein in $20 \mathrm{mM}$ Tris- $\mathrm{HCl}, \mathrm{pH} 7.7,40 \mathrm{mM} \mathrm{NaCl}, 5 \mathrm{mM} \mathrm{BME}$, and $0.2 \mathrm{mM}$ EDTA) with $1 \mu \mathrm{L}$ reservoir solution containing $15 \%$ PEG $8000,0.2 \mathrm{M}$ calcium acetate and $0.1 \mathrm{M}$ sodium cocadylate. The crystals were flash frozen after soaking in reservoir solution supplemented with $15 \%(w / v)$ glycerol. A MAD data set at three different wavelengths $\left(\lambda_{\text {inflection }}=0.9796 \AA, \lambda_{\text {peak }}=0.9794 \AA, \lambda_{\text {remote }}=\right.$ $0.9600 \AA$ ) was collected with a selenomethionyl human elF2B $\varepsilon$-CTD crystal at $100 \mathrm{~K}$ on beamline X12C, NSLS, Brookhaven National Laboratory. A 2.0-Å resolution data set from another crystal was collected at wavelength $0.9794 \AA$ for refinement at $100 \mathrm{~K}$ on beamline BL17U1 of Shanghai Synchrotron Radiation Facility. All the data were processed with HKL2000 (Otwinowski and Minor, 1997). The crystal belonged to the space group centered orthorhombic $C 222_{1}$ and contained one human elF2B -CTD molecule in the asymmetric unit $\left(V_{m}=2.47 \AA^{3} \mathrm{Da}^{-1}\right.$, solvent content $\left.=50.2 \%\right)$. Five of the six expected selenium atoms were located and used for phase determination at 2.3- $\AA$ by SOLVE (Terwilliger and Berendzen, 1999), and phases were 


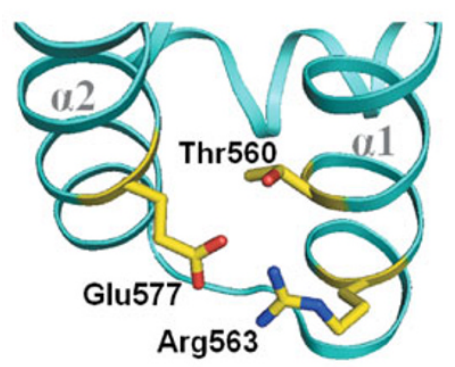

$\mathrm{C}$

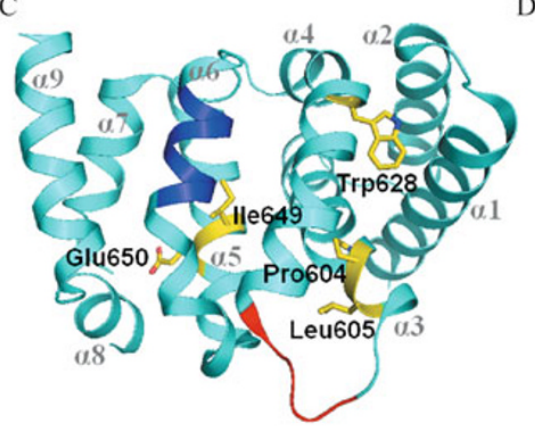

$\mathrm{E}$

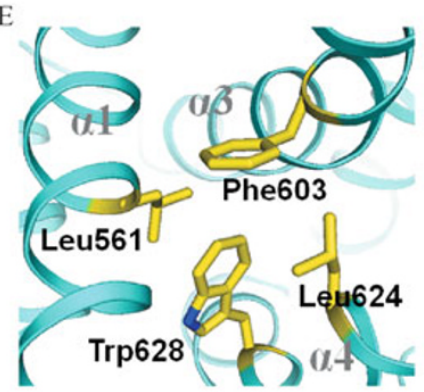

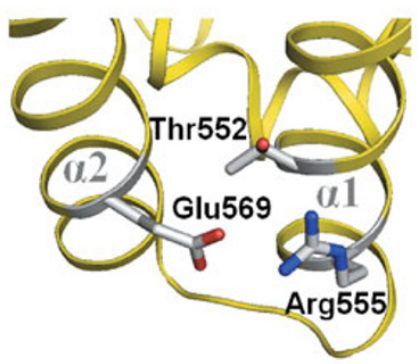

$\mathrm{D}$

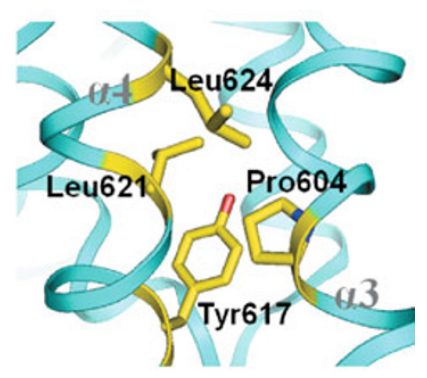

$\mathrm{F}$

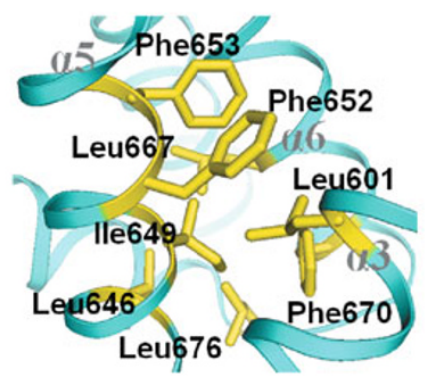

G

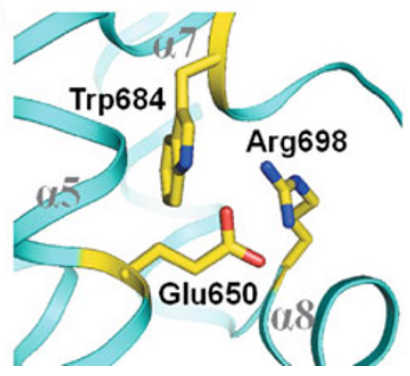

Figure 4. The critical residues of human elF2B $\varepsilon$-CTD concerned with GEF activity of elF2B and VWM/CACH disease. (A)

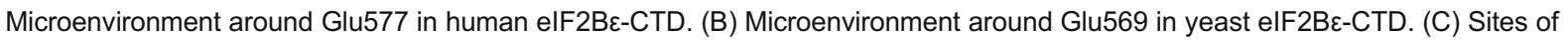
mutations related to VWM disease are labeled on the structure of human elF2BE-CTD. Deletion sites of Ser610-Asp613 $\Delta$ and Lys665-Tyr671 $\Delta$ are colored red and blue, respectively. Other mutation sites at Pro604, Leu605, Trp628, lle649 and Glu650 are labeled in yellow. (D) Hydrophobic contacts of Pro604 with surrounding residues Tyr617, Leu621 and Leu624. (E) Hydrophobic contacts of Trp628 with surrounding residues Leu561, Leu624 and Phe603. (F) Hydrophobic core formed by Ile649, Leu601, Leu652, Phe653, Leu667, Leu646, Leu676 and Phe670. (G) The ion pair and hydrogen bond formed by Glu650 with Arg698 and Trp684, respectively.

subsequently improved by density modification with RESOLVE (Terwilliger, 2000, 2003) for initial model building. The model was refined with 2.0- $\AA$ resolution data by Coot (Emsley and Cowtan,
2004), CNS (Brunger, 1998, 2007) and Refmac5 (Murshudov et al., 1997) for additional model building and adjustment. In the final model, the electron densities of residues Glu716-Asp721 were invisible and 
those of Glu715, Thr690 and Thr691 were poor, so these 9 residues were excluded.

\section{Structure modeling and analysis}

In the experimental model, the incidental mutation Glu678Gly was located at the macromolecular surface. Therefore, it was expected to make the least impact on the 3-D folding of human elF2BE-CTD. To achieve a realistic distribution of electrostatic potential, structure modeling was performed to replace Gly678 by Glu, using Modeler (Sali and Blundell, 1993; Fiser et al., 2000; Martí-Renom et al., 2000; Eswar et al., 2006). As expected, only a limited change was observed in the adjusted model, where the side chain of Glu714 was slightly altered. Based on the adjusted structure, the surface electrostatic property was analyzed with APBS (Baker et al., 2001) and structural representations were generated using PyMOL [http://www.pymol. org].

\section{COORDINATES DEPOSITION}

The atomic coordinates and structural factors for human elF2BE-CTD have been deposited in the Protein Data Bank (PDB) with the accession code 3JUI.

\section{ACKNOWLEDGEMENTS}

The authors would like to thank Prof. Ruiming $\mathrm{Xu}$ in Institute of Biophysics, Chinese Academy of Sciences for MAD data collection at the National Synchrotron Light Source, Brookhaven National Laboratory (New York, USA). Other synchrotron-radiation experiments were performed at BL17U1 of Shanghai Synchrotron Radiation Facility (SSRF, Shanghai, China). This work was supported by the National Programs for High Technology Research and Development Program (863 Program) (Grant No. 2006AA02A316) and the National Basic Research Program (973 Program) (Grant Nos. 2004CB520801, 2006CB910903, 2007CB914304, 2009CB825501 and 2010CB912301) of the Ministry of Science and Technology, National Natural Science Foundation of China (Grant Nos. 30721003 and 30870484), and the Chinese Academy of Sciences (Grant No. KSCX2-YW-R61).

\section{ABBREVIATIONS}

elF2B, eukaryotic translation initiation factor 2B; elF2B-CTD, Cterminal domain of human elF2B; GEF, guanine nucleotide exchange factor; PIC, pre-initiation complex; VWM, vanishing white matter; $\eta 1$, the first $3_{10}$-helix; $\eta 2$, the second $3_{10}$-helix

\section{REFERENCES}

Asano, K., Krishnamoorthy, T., Phan, L., Pavitt, G.D., and Hinnebusch, A.G. (1999). Conserved bipartite motifs in yeast elF5 and elF2Bepsilon, GTPase-activating and GDP-GTP exchange factors in translation initiation, mediate binding to their common substrate elF2. EMBO J 18, 1673-1688.

Baker, N.A., Sept, D., Joseph, S., Holst, M.J., and McCammon, J.A. (2001). Electrostatics of nanosystems: application to microtubules and the ribosome. Proc Natl Acad Sci U S A 98, 10037-10041.
Bieniossek, C., Schütz, P., Bumann, M., Limacher, A., Uson, I., and Baumann, U. (2006). The crystal structure of the carboxy-terminal domain of human translation initiation factor elF5. J Mol Biol 360, 457-465.

Boesen, T., Mohammad, S.S., Pavitt, G.D., and Andersen, G.R. (2004). Structure of the catalytic fragment of translation initiation factor $2 \mathrm{~B}$ and identification of a critically important catalytic residue. J Biol Chem 279, 10584-10592.

Brunger, A.T. (2007). Version 1.2 of the Crystallography and NMR system. Nat Protoc 2, 2728-2733.

Brunger, A.T., Adams, P.D., Clore, G.M., DeLano, W.L., Gros, P., Grosse-Kunstleve, R.W., Jiang, J.S., Kuszewski, J., Nilges, M., Pannu, N.S., et al. (1998). Crystallography \& NMR system: a new software suite for macromolecular structure determination. Acta Crystallogr 54, 905-921.

Cigan, A.M., Bushman, J.L., Boal, T.R., and Hinnebusch, A.G. (1993). A protein complex of translational regulators of GCN4 mRNA is the guanine nucleotide-exchange factor for translation initiation factor 2 in yeast. Proc Natl Acad Sci U S A 90, 5350-5354.

Das, S., Maiti, T., Das, K., and Maitra, U. (1997). Specific interaction of eukaryotic translation initiation factor 5 (elF5) with the beta-subunit of elF2. J Biol Chem 272, 31712-31718.

Das, S., and Maitra, U. (2000). Mutational analysis of mammalian translation initiation factor 5 (elF5): role of interaction between the beta subunit of elF2 and elF5 in elF5 function in vitro and in vivo. Mol Cell Biol 20, 3942-3950.

Dever, T.E. (2002). Gene-specific regulation by general translation factors. Cell 108, 545-556.

Emsley, P., and Cowtan, K. (2004). Coot: model-building tools for molecular graphics. Acta Crystallogr 60, 2126-2132.

Eswar, N., Webb, B., Marti-Renom, M.A., Madhusudhan, M.S., Eramian, D., Shen, M.Y., Pieper, U., and Sali, A. (2006). Comparative protein structure modeling using Modeller. Curr Protoc Bioinformatics Chapter 5, Unit 5.6.

Fiser, A., Do, R.K., and Sali, A. (2000). Modeling of loops in protein structures. Protein Sci 9, 1753-1773.

Fogli, A., and Boespflug-Tanguy, O. (2006). The large spectrum of elF2B-related diseases. Biochem Soc Trans 34, 22-29.

Gomez, E., Mohammad, S.S., and Pavitt, G.D. (2002). Characterization of the minimal catalytic domain within elF2B: the guaninenucleotide exchange factor for translation initiation. EMBO J 21, 5292-5301.

Gouet, P., Courcelle, E., Stuart, D.I., and Métoz, F. (1999). ESPript: analysis of multiple sequence alignments in PostScript. Bioinformatics 15, 305-308.

Hinnebusch, A.G. (2005). Translational regulation of GCN4 and the general amino acid control of yeast. Annu Rev Microbiol 59, 407-450.

Holm, L., and Park, J. (2000). DaliLite workbench for protein structure comparison. Bioinformatics 16, 566-567.

Kapp, L.D., and Lorsch, J.R. (2004). The molecular mechanics of eukaryotic translation. Annu Rev Biochem 73, 657-704.

Krishnamoorthy, T., Pavitt, G.D., Zhang, F., Dever, T.E., and Hinnebusch, A.G. (2001). Tight binding of the phosphorylated alpha subunit of initiation factor 2 (elF2alpha) to the regulatory subunits of guanine nucleotide exchange factor elF2B is required for inhibition of translation initiation. Mol Cell Biol 21, 5018-5030. 
Larkin, M.A., Blackshields, G., Brown, N.P., Chenna, R., McGettigan, P.A., McWilliam, H., Valentin, F., Wallace, I.M., Wilm, A., Lopez, R., et al. (2007). Clustal $W$ and Clustal $X$ version 2.0. Bioinformatics 23, 2947-2948.

Li, W., Wang, X., Van Der Knaap, M.S., and Proud, C.G. (2004). Mutations linked to leukoencephalopathy with vanishing white matter impair the function of the eukaryotic initiation factor $2 \mathrm{~B}$ complex in diverse ways. Mol Cell Biol 24, 3295-3306.

Maletkovic, J., Schiffmann, R., Gorospe, J.R., Gordon, E.S., Mintz, M., Hoffman, E.P., Alper, G., Lynch, D.R., Singhal, B.S., Harding, C., et al. (2008). Genetic and clinical heterogeneity in elF2Brelated disorder. J Child Neurol 23, 205-215.

Marcotrigiano, J., Lomakin, I.B., Sonenberg, N., Pestova, T.V., Hellen, C.U., and Burley, S.K. (2001). A conserved HEAT domain within elF4G directs assembly of the translation initiation machinery. Mol Cell 7, 193-203.

Marintchev, A., and Wagner, G. (2004). Translation initiation: structures, mechanisms and evolution. Q Rev Biophys 37, 197-284.

Martí-Renom, M.A., Stuart, A.C., Fiser, A., Sánchez, R., Melo, F., and Sali, A. (2000). Comparative protein structure modeling of genes and genomes. Annu Rev Biophys Biomol Struct 29, 291-325.

Mohammad-Qureshi, S.S., Haddad, R., Hemingway, E.J., Richardson, J.P., and Pavitt, G.D. (2007a). Critical contacts between the eukaryotic initiation factor 2B (elF2B) catalytic domain and both elF2beta and-2gamma mediate guanine nucleotide exchange. Mol Cell Biol 27, 5225-5234.

Mohammad-Qureshi, S.S., Haddad, R., Palmer, K.S., Richardson, J. P., Gomez, E., and Pavitt, G.D. (2007b). Purification of FLAGtagged eukaryotic initiation factor 2B complexes, subcomplexes, and fragments from Saccharomyces cerevisiae. Methods Enzymol 431, 1-13.

Mohammad-Qureshi, S.S., Jennings, M.D., and Pavitt, G.D. (2008). Clues to the mechanism of action of elF2B, the guaninenucleotide-exchange factor for translation initiation. Biochem Soc Trans 36, 658-664.

Murshudov, G.N., Vagin, A.A., and Dodson, E.J. (1997). Refinement of macromolecular structures by the maximum-likelihood method. Acta Crystallogr 53, 240-255.

Otwinowski, Z., and Minor, W. (1997). Processing of X-ray diffraction data collected in oscillation mode. Methods Enzymol 276, 307-326.

Pavitt, G.D. (2005). elF2B, a mediator of general and gene-specific translational control. Biochem Soc Trans 33, 1487-1492.

Pavitt, G.D., Ramaiah, K.V., Kimball, S.R., and Hinnebusch, A.G. (1998). elF2 independently binds two distinct elF2B subcomplexes that catalyze and regulate guanine-nucleotide exchange. Genes
Dev 12, 514-526.

Sali, A., and Blundell, T.L. (1993). Comparative protein modelling by satisfaction of spatial restraints. J Mol Biol 234, 779-815.

Scali, O., Di Perri, C., and Federico, A. (2006). The spectrum of mutations for the diagnosis of vanishing white matter disease. Neurol Sci 27, 271-277.

Singh, C.R., Lee, B., Udagawa, T., Mohammad-Qureshi, S.S., Yamamoto, Y., Pavitt, G.D., and Asano, K. (2006). An elF5/elF2 complex antagonizes guanine nucleotide exchange by elF2B during translation initiation. EMBO J 25, 4537-4546.

Smirnova, J.B., Selley, J.N., Sanchez-Cabo, F., Carroll, K., Eddy, A. A., McCarthy, J.E., Hubbard, S.J., Pavitt, G.D., Grant, C.M., and Ashe, M.P. (2005). Global gene expression profiling reveals widespread yet distinctive translational responses to different eukaryotic translation initiation factor 2B-targeting stress pathways. Mol Cell Biol 25, 9340-9349.

Sonenberg, N., and Hinnebusch, A.G. (2007). New modes of translational control in development, behavior, and disease. Mol Cell 28, 721-729.

Terwilliger, T.C. (2000). Maximum-likelihood density modification. Acta Crystallogr 56, 965-972.

Terwilliger, T.C. (2003). Automated main-chain model building by template matching and iterative fragment extension. Acta Crystallogr 59, 38-44.

Terwilliger, T.C., and Berendzen, J. (1999). Automated MAD and MIR structure solution. Acta Crystallogr 55, 849-861.

Wang, X., Paulin, F.E., Campbell, L.E., Gomez, E., O'Brien, K., Morrice, N., and Proud, C.G. (2001). Eukaryotic initiation factor 2B: identification of multiple phosphorylation sites in the epsilonsubunit and their functions in vivo. EMBO J 20, 4349-4359.

Wang, X., and Proud, C.G. (2008). A novel mechanism for the control of translation initiation by amino acids, mediated by phosphorylation of eukaryotic initiation factor 2B. Mol Cell Biol 28, 1429-1442.

Wei, Z., Xue, Y., Xu, H., and Gong, W. (2006). Crystal structure of the C-terminal domain of S.cerevisiae elF5. J Mol Biol 359, 1-9.

Williams, D.D., Pavitt, G.D., and Proud, C.G. (2001a). Characterization of the initiation factor elF2B and its regulation in Drosophila melanogaster. J Biol Chem 276, 3733-3742.

Williams, D.D., Price, N.T., Loughlin, A.J., and Proud, C.G. (2001b). Characterization of the mammalian initiation factor elF2B complex as a GDP dissociation stimulator protein. J Biol Chem 276, 24697-24703.

Wu, Y., Pan, Y., Du, L., Wang, J., Gu, Q., Gao, Z., Li, J., Leng, X., Qin, J., Wu, X., et al. (2009). Identification of novel EIF2B mutations in Chinese patients with vanishing white matter disease. J Hum Genet 54, 74-77. 